\title{
Eliminación de contaminantes orgánicos en agua empleando nanomateriales catalíticos fotoactivos
}

\section{Elimination of organic pollutants in water using photoactive} nanomaterials.

Doctorando:

\author{
Ariel José Vinuesa \\ Centro de Investigación y Tecnología Química UTN-CONICET \\ arielvinuesa@gmail.com
}

Directora: Dra. Clara Saux Co-Directora: Dra. María Soledad Renzini

\begin{abstract}
Resumen
La producción de agentes químicos contaminantes provenientes de actividades industriales, agrícolas y domésticas, como también sus emisiones, son algunos de los problemas medioambientales que están ganando cada vez más relevancia. Debido al crecimiento demográfico y el consecuente aumento de la actividad antropogénica, los cursos de agua constituyen uno de los recursos naturales más perjudicados. Como consecuencia, el medio ambiente y la calidad de vida de los habitantes del entorno se ven gravemente afectados. Cabe destacar que en los últimos años se ha detectado la presencia de ciertos contaminantes, tales como fármacos o pesticidas, en bajas concentraciones aún en el agua tratada. A los mismos se los denomina Contaminantes Emergentes puesto que su presencia ha sido detectada recientemente gracias al avance en las técnicas analíticas, pero cuyo consumo regular puede provocar serios problemas a la salud y al ambiente y que las tecnologías de purificación de aguas actualmente empleadas no son eficientes para su eliminación.

En este contexto, los procesos de oxidación avanzada (POA) aparecen como una solución eficiente y complementaria a los procesos y tecnologías existentes. El presente plan de tesis tiene el objetivo de sintetizar y evaluar materiales microporosos, concretamente zeolitas sintéticas (Y, Beta y ZSM-5) y naturales, modificados con metales de transición (Fe, Zn, Sn, etc.) para ser usados en la eliminación fotocatalítica de contaminantes orgánicos presentes en los cursos de agua, tales como drogas farmacológicas (levamisol), pesticidas (glifosato) y colorantes industriales (naranja de metilo, alizarina, etc.).
\end{abstract}

Palabras claves: Fotocatálisis, Contaminantes Emergentes, Zeolitas, Procesos de Oxidación Avanzada.

\begin{abstract}
The production of chemical pollutants from industrial, agricultural and domestic activities, as well as their emissions, is one of the most relevant and important environmental problems. Waterways are one of the most affected natural resources due to demographic growth and the consequent increase in anthropogenic activity. Subsequently, the environment and the quality of life of the surrounding inhabitants are severely affected.

In this context, advanced oxidation processes (APOs) appear as an efficient and complementary solution to the existing processes and technologies. This work aims to synthesize and evaluate microporous materials, namely synthetic Y, Beta and ZSM-5 and natural zeolites, and to modify them with transition metals (Fe, Zn, Sn, etc.) in order to be used in the photocatalytic degradation of organic pollutants present in water courses, such as pharmacological drugs (levamisole), pesticides (glyphosate) and industrial dyes (methyl orange, alizarin, etc) .
\end{abstract}

Keywords: Photocatalysis, Emerging Pollutants, Zeolites, Advanced Oxidation Processes. 


\section{Introducción}

De los múltiples contaminantes que pueden encontrarse en los cursos acuosos, los colorantes son de origen antropogénico y su presencia está asociada a los efluentes de las industrias textiles, de papel, cuero, plásticos, entre otras. Este tipo de impurezas reducen la penetración de la luz en los ecosistemas acuáticos y puede llegar a afectar la calidad del agua de consumo humano [1]. En particular, aquellos colorantes de tipo azoico (que constituyen el 70\% de los colorantes aplicados en la actualidad) presentan una gran dificultad para su remoción por los métodos convencionales, tales como floculación, sedimentación o adsorción, debido a su compleja estructura y elevada estabilidad [2]. Ya que los colorantes industriales representan un grupo de contaminantes de suma importancia para los cursos de agua y que el seguimiento su degradación puede ser cuantificado fácilmente por espectroscopia UV-Vis, se comenzaron los estudios de evaluación de los catalizadores eligiendo al naranja de metilo (NM) como contaminante modelo. Es un colorante azoico (ácido 4-((4Dimetilamino)fenil)azo)benzenosulfónico) empleado habitualmente en preparaciones farmacéuticas, teñido de telas y plásticos, etc.

Dentro de los potenciales catalizadores para este tipo de reacciones que involucran el empleo de luz, los nanocristales de $\alpha-\mathrm{Fe}_{2} \mathrm{O}_{3}$ (hæmatita) presentan la ventaja de poder sintetizarse con una selectividad de forma que potencia su actividad. La hæmatita es un semiconductor de bajo costo y con un band gap de $2,2 \mathrm{eV}$, ideal para la eliminación fotocatalítica de contaminantes por su sensibilidad a la luz visible y amigabilidad con el ambiente [3]. Además, el hierro puede reaccionar con $\mathrm{H}_{2} \mathrm{O}_{2}$ para dar lugar a radicales hidroxilo que poseen un elevado potencial de oxidación. En fase homogénea, la reacción con $\mathrm{Fe}^{2+}$ es muy violenta y puede ser potenciada utilizando luz. Sin embargo, la reacción se lleva a cabo en un estrecho rango de pH $(2,5-4)$, requiere la reposición del precursor de hierro ya que éste forma hidróxidos insolubles que a su vez constituyen un residuo sólido añadido al proceso. Fases de óxidos de hierro como $\alpha-\mathrm{Fe}_{2} \mathrm{O}_{3}$ son capaces de llevar a cabo la reacción Fenton y Foto-Fenton en fase heterogénea salvando los inconvenientes de la catálisis homogénea.

Se modificaron con hierro tres matrices zeoltíticas, H-ZSM-5, H-ZSM-5 con porosidad jerarquizada y zeolitas naturales provenientes de una cantera sanjuanina. Las modificaciones se realizaron mediante impregnación en húmedo, intercambio iónico y por síntesis hidrotermal empleando diferentes precursores del metal. Además, se sintetizaron dodecaedros truncados de $\alpha-\mathrm{Fe}_{2} \mathrm{O}_{3}$ por vía hidrotermal. Todos estos materiales fueron evaluados en la fotodegradación de naranja de metilo y caracterizados mediante DRX, DRS- UV-Vis, FT-IR, TPR, SEM-EDS, TEM.

Las zeolitas naturales son atractivas cuando se tiene en cuanta que son muy abundantes en la naturaleza. Si estos materiales pueden ser adaptados a los procesos en los cuales se utilizan zeolitas o soportes inorgánicos de síntesis similares, no solo representarían una importante reducción en los costos y energía de producción, sino también un aporte significativo desde el punto de vista de la química verde. La producción industrial de zeolitas utiliza moléculas orgánicas altamente contaminantes además de altas presiones y temperaturas. No obstante, los materiales naturales son mucho menos uniformes en composición, más lábiles y acotados en cuanto a estructuras cristalinas. Paralelamente al uso de las zeolitas sintéticas, se trabaja con muestras de clinoptilolita obtenidas de una cantera sanjuanina.

Los materiales sintetizados se evaluaron en un sistema de reacción que consistió en un fotorreactor anular de vidrio Pyrex, agitado magnéticamente, con una lámpara de mercurio $125 \mathrm{~W}$ de alta presión y un aireador. Se partió de una solución $8.10^{-6} \mathrm{M}$ de naranja de metilo (NM). Se consideró como tiempo inicial de reacción al momento en el que se incorporó el catalizador $(200 \mathrm{mg})$, junto con el aireador, la lámpara y la cantidad correspondiente de $\mathrm{H}_{2} \mathrm{O}_{2}$. El volumen de solución empleado fue de $400 \mathrm{ml}$. En forma periódica se tomaron muestras de la solución reaccionante las cuales fueron filtradas, para separar el catalizador, y analizadas en un Espectrofotómetro UV-Vis realizando un barrido entre 200 y $600 \mathrm{~nm}$. Además, se realizó un seguimiento de los cambios de $\mathrm{pH}$ y temperatura.

\section{Resultados}

\section{Caracterización de los materiales}

Los patrones de difracción de rayos X (Figura 1) muestran que la estructura MFI de las zeolitas utilizadas como soporte no sufrió alteraciones luego del tratamiento hidrotermal para la incorporación del hierro. En ZFe se observan incrementos en los ángulos correspondientes a las reflexiones características de $\alpha-\mathrm{Fe}_{2} \mathrm{O}_{3}$, mientras que los mismos no son distinguibles en $\mathrm{ZA}-\mathrm{Fe}$, lo que sugeriría que las partículas son pequeñas con una alta dispersión en la superficie del soporte. Los espectros de DRS UV-Vis medidos (Figura 2), fueron convertidos a la función de Kubelka-Munk con respecto a la longitud de onda. El espectro se dividió en tres 
secciones que corresponden a tres especies de hierro diferentes [6]. Hasta los $280 \mathrm{~nm}$ las bandas corresponden a iones de $\mathrm{Fe}^{3+}$ aislados. Entre 280 y $450 \mathrm{~nm}$ se observan bandas asociadas a pequeñas especies oligonucleares de óxido de hierro $\mathrm{Fe}^{3+}{ }_{\mathrm{x}} \mathrm{O}_{\mathrm{y}}$, mientras que a longitudes mayores de $450 \mathrm{~nm}$ aparecen las bandas características de $\alpha-\mathrm{Fe}_{2} \mathrm{O}_{3}$ [7]. ZA-Fe posee un menor aporte por parte de la fracción correspondiente a $\alpha$-Fe2O3 que Z-Fe. Esto puede ser explicado en base a las imágenes SEM, en donde se pueden distinguir claramente cristales de $\alpha-\mathrm{Fe}_{2} \mathrm{O}_{3}$ sobre los cristales de Z, no así en ZA, al igual que en los patrones de DRX de ambos materiales.

Las imágenes SEM (Figura $3 \mathrm{~d}$ ) confirman la existencia de partículas de un tamaño promedio de 66,25 \pm 8,6 $\mathrm{nm}$ sobre los cristales de Z. El análisis de EDS señala la presencia de hierro en esta muestra, por lo que junto con los resultados de DRX concluimos que se trata de nanopartículas de hematita. Por su parte, ZA (Figura $3 \mathrm{c}$ ) se ve prácticamente inalterada por el tratamiento hidrotermal en las micrografías. No se observa la aparición de clusters de óxidos o cristales, más allá de los característicos de la estructura MFI. Sin embargo, el análisis de EDS indica que dicho material contiene hierro.

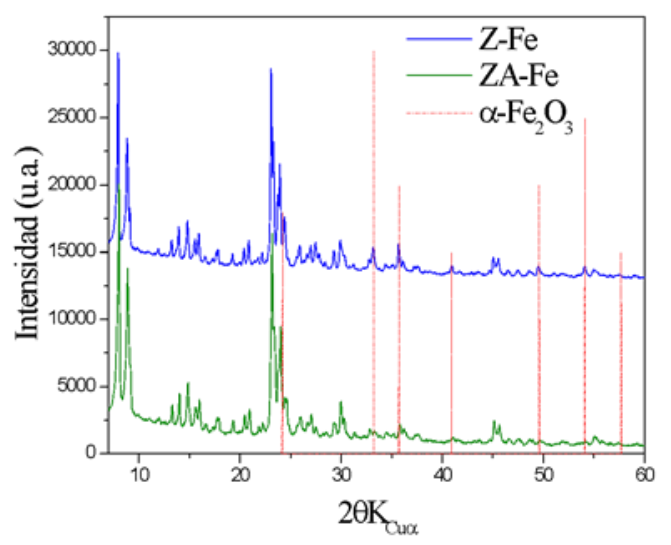

Figura 1. Patrones de difracción de los catalizadores evaluados.

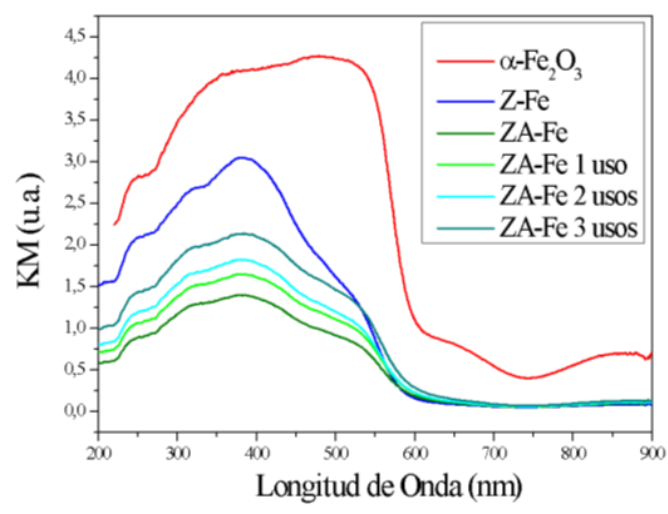

Figura 2. Espectros DRS UV-Vis de los catalizadores frescos y después de la reacción.
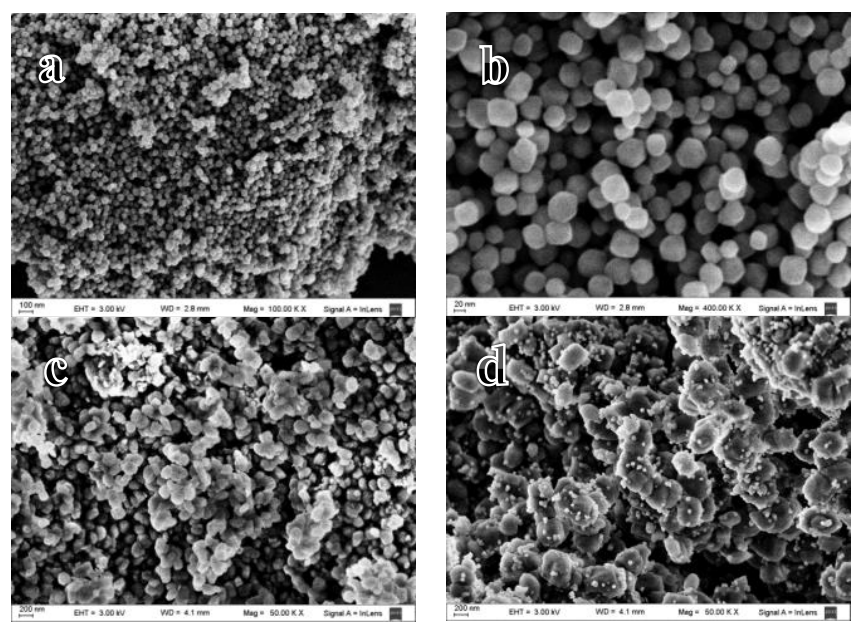

Figura 3. Micrografías de barrido electrónico. a) y b) Nanopartículas de $\alpha-\mathrm{Fe}_{2} \mathrm{O}_{3}$, c) ZA-Fe y d) Z-Fe. 


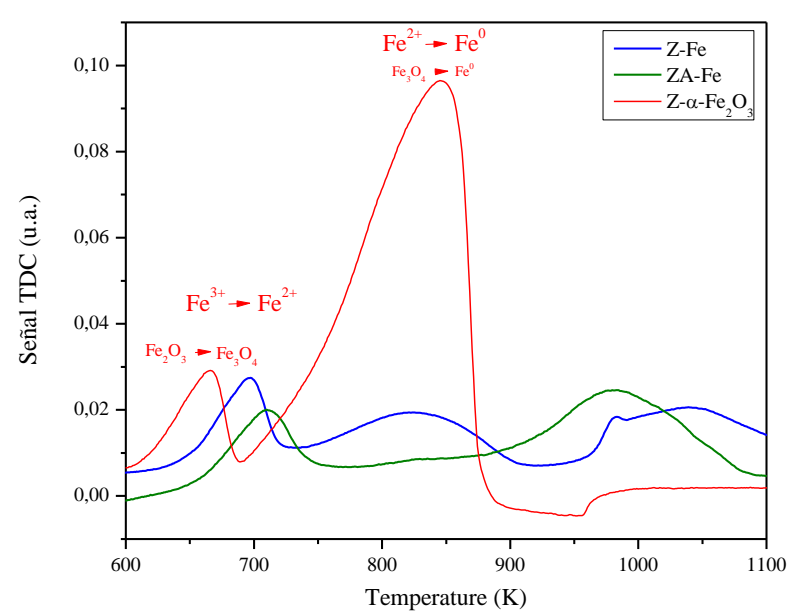

Figura 4. Perfiles de reducción a temperatura programada de Z-Fe, ZA-Fe y la mezcla mecánica Z- $\alpha-\mathrm{Fe}_{2} \mathrm{O}_{3}$

Los perfiles de reducción a temperatura programada de las muestras se presentan en la Figura 4. En los mismos se observa también la diferencia entre las especies de hierro incorporadas en $\mathrm{Z}$ y ZA. Se analizaron tres muestras, ZA-Fe, Z-Fe y una mezcla mecánica de $\alpha-\mathrm{Fe}_{2} \mathrm{O}_{3}$ y Z (Z- $\left.\alpha-\mathrm{Fe}_{2} \mathrm{O}_{3}\right)$. El perfil de Z- $\alpha-\mathrm{Fe}_{2} \mathrm{O}_{3}$ se empleó como referencia para la interpretación de los de ZA-Fe y Z-Fe. El perfil de la mezcla mecánica muestra dos picos bien diferenciables, por lo tanto asumimos que la reducción se llevó a cabo en dos pasos hasta hierro metálico. El primero corresponde a la reducción de hematita a magnetita $3 \alpha-\mathrm{Fe}_{2} \mathrm{O}_{3}+\mathrm{H}_{2} \rightarrow 2 \mathrm{Fe}_{3} \mathrm{O}_{4}+\mathrm{H}_{2} \mathrm{O}$. Mientras que el segundo pico representa la reducción completa a hierro metálico a partir de magnetita $\mathrm{Fe}_{3} \mathrm{O}_{4}+4 \mathrm{H}_{2} \rightarrow 3 \mathrm{Fe}^{0}$ $+4 \mathrm{H}_{2} \mathrm{O}$. De acuerdo con estas ecuaciones, el primer pico debería representar el $11 \%$ del consumo total de hidrógeno y el segundo el 89\% [10]. Los cálculos de área obtenidos de la integración de la curva de Z- $\mathrm{Fe}_{3} \mathrm{O}_{4}$ arrojan valores de $9 \%$ y $91 \%$ para los respectivos picos. Los perfiles obtenidos para ZA-Fe y particularmente ZFe presentan diferencias con respecto a Z $-\alpha-\mathrm{Fe}_{2} \mathrm{O}_{3}$. La asignación específica de cada pico observado en la señal del TCD a una especie de hierro es una tarea compleja, sin embargo, algunas observaciones genearles se pueden realizar en base a los gráficos obtenidos. Tanto en ZA-Fe como Z-Fe, las señales de consumo de H2 se desplazaron a temperaturas mayores. Esto es esperable para un óxido soportado, ya que las interacciones con el soporte dificultan la reducción. Además, podemos ver que la muestra ZA-Fe es más simple en cuanto a las especies que la componen ya que tiene básicamente sólo dos picos, al igual que Z- $\alpha-\mathrm{Fe}_{2} \mathrm{O}_{3}$. En cambio el perfil de Z-Fe es más complejo y podemos suponer que es un reflejo de una composición más compleja en cuanto a las especies de hierro incorporadas en el material. Los nuevos picos a alrededor de $980 \mathrm{~K}$ podrían asignarse a la reducción de $\mathrm{FeO}[8]$, un intermediario esperado en la reducción de $\mathrm{Z}-\alpha-\mathrm{Fe}_{2} \mathrm{O}_{3}$. Incluso podría tratarse de iones $\mathrm{Fe}^{3+}$ incorporados isomórficamente en la red zeolítica luego del tratamiento hidrotermal $[9,12]$.

\section{Actividad catalítica}

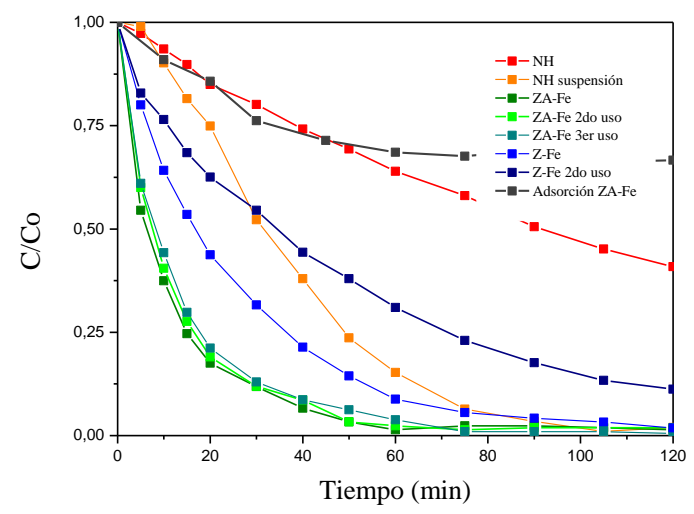

Figura 5. Actividad catalítica para la fotodegradación Fenton de NM.

La Figura 5 muestra los resultados obtenidos del análisis de todas las reacciones realizadas en un lapso de $120 \mathrm{~min}$. En cada caso se registraron los cambios de temperatura y $\mathrm{pH}$ sin intervención, siendo de alrededor de 24 a $31^{\circ} \mathrm{C}$ y de $5,67(\mathrm{pH}$ de la solución de $\mathrm{NM}$ ) a 4,20 respectivamente. Todos los materiales evaluados 
presentaron actividad frente a la degradación de NM. La prueba de adsorción se llevó a cabo con ZA-Fe y como resultado se observó una disminución en la concentración de la solución de NM del 34\%. En el caso de las nanopartículas $(\mathrm{NH})$, se realizaron pruebas con el material seco luego del proceso de síntesis. Las mismas presentaron los resultados menos satisfactorios. Presumiendo que la falta de actividad podría deberse a la aglomeración irreversible de las nanopartículas [11]. A fin de superar este inconveniente, se repitieron las evaluaciones, pero utilizando una cantidad equivalente del material sin secar. Para ello se incorporaron al sistema de reacción las nanopartículas en suspensión obtenidas luego del lavado post-síntesis. La actividad se incrementó notablemente, por lo que se puede inferir que la aglomeración de las nanopartículas es un factor que limita la actividad de estos materiales. Con el objeto de incrementar la actividad de las nanopartículas de $\alpha$ $\mathrm{Fe}_{2} \mathrm{O}_{3}$ (NH) y facilitar su recuperación, se evaluaron los materiales soportados sobre zeolitas ZSM-5 microporosas (Z-Fe) y jerarquizadas (ZA-Fe). Con Z-Fe se obtuvo una completa eliminación de NM al cabo de 120 min de reacción. Sin embargo, al filtrar la mezcla de reacción, la misma presentaba coloración debida a la pérdida de nanopartículas en el medio de reacción. El material recuperado se lavó con agua ultrapura y se secó en estufa a $120^{\circ} \mathrm{C}$ durante $48 \mathrm{~h}$. Luego se reutilizó en una segunda reacción, en la cual la velocidad de degradación disminuyó considerablemente como consecuencia del lixiviado de las NH. No obstante, el 90\% de decoloración se obtuvo a los 120 min de reacción. ZA-Fe mostró la mayor actividad frente a la decoloración foto-Fenton del NM. En $60 \mathrm{~min}$ la solución fue completamente decolorada. El material fue recuperado y reutilizado al igual que Z-Fe. La actividad disminuyó levemente después de 3 usos (la decoloración completa se alcanzó a los $70 \mathrm{~min}$ ). Las muestras del catalizador recuperado fueron analizadas por DRS UV-Vis luego de cada uso (Figura 2). Como se observa en los espectros, se registró un aumento constante en la absorción del material usado, con respecto al original. Sabiendo que los iones $\mathrm{Fe}^{2+}$ no son activos en DRS UV-Vis, es posible asignar este aumento en la intensidad a la oxidación de estos cationes durante la reacción y consecuentemente un incremento de las especies de $\mathrm{Fe}^{3+}[6]$.

\section{Zeolitas naturales}

Las muestras de clinoptilolita fueron caracterizadas mediante DRX para evaluar la naturaleza de las fases que componían el material. En base a los resultados obtenidos, se procedió a realizar procesos de modificación para eliminar fases indeseadas y liberar la estructura microporosa. En la figura 6 se resumen algunos de los resultados tanto de la modificación como de uso de los catalizadores obtenidos. En la Figura 6 A, se puede observar el incremento del área de una misma muestra de clinoptilolita a medida que se expone a concentraciones más elevadas de ácido (Z-CMA es la muestra sin tratamiento y Z-CMA-02, Z-CMA-05 y Z-CMA15 corresponden a muestras sometidas a un lavado con ácido nítrico en orden creciente de acidéz) de $10 \mathrm{~m}^{2} / \mathrm{g}$ en el material natural a $152 \mathrm{~m}^{2} / \mathrm{g}$ luego del tratamiento ácido. En la figura 6, B, podemos observar los patrones de difracción de una muestra natural y luego del tratamiento. La muestra natural contiene carbonato de calcio, el cuál es completamente eliminado luego del tratamiento ácido. Los catalizadores obtenidos a partir de estos materiales están siendo evaluados no sólo en la fotodegradación de contaminantes, sino también en otras aplicaciones. Junto a Heredia et al. [13] se evaluó la capacidad de remoción de As(III) de una serie de catalizadores obtenidos de la modificación de clinoptilolita con Fe (III), figura 6, C.
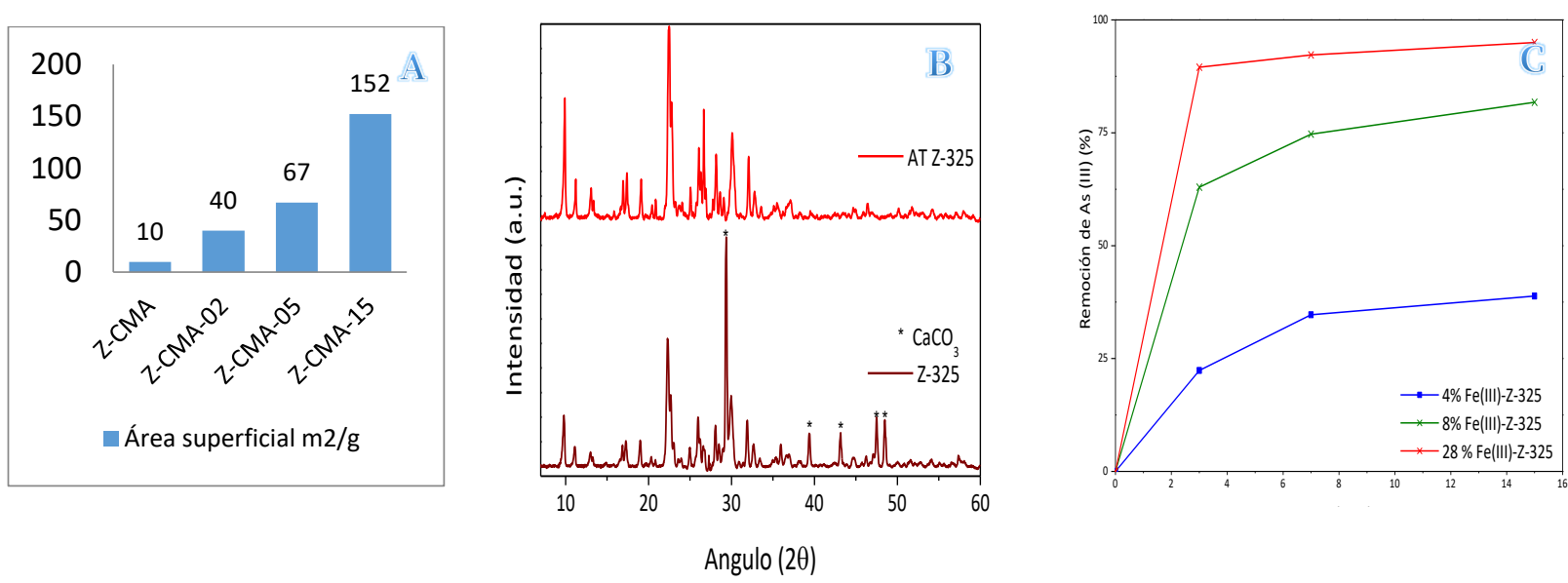

Figura 6. A) Incremento de área superficial con tratamiento ácido. B) DRX de zeolita natural antes y después de tratamiento ácido. C) Remoción de As(III) en solución acuosa. 


\section{Conclusiones}

Se sintetizaron materiales con hierro en diferentes formas como especie activa en la degradación fotoFenton de un colorante azoico, naranja de metilo. Por un lado se logró obtener nanopartículas de hematita con una especificidad de forma muy elevada, tanto por la dispersión de tamaño de partícula, como por la morfología de los cristales. Luego se modificó la síntesis hidrotermal de las nanopartículas con la adición de dos matrices zeolíticas con la intención de soportarlas. Todos los materiales dieron resultados prometedores para ser utilizados como catalizadores foto-Fenton en la degradación de contaminantes orgánicos.

Es indudable la importancia que tiene el método de incorporación y la naturaleza de las interacciones soporte/especie activa sobre la actividad final del catalizador. Más estudios de caracterización se realizarán para tratar de determinar con más certeza cuáles son las especies más activas y dónde están alojadas dentro de las zeolitas. Una vez determinado esto, se procederá a hacer ensayos con las matrices naturales modificadas y contaminantes de otra naturaleza como los mencionados anteriormente.

\section{Referencias}

[1] Mandal, S., Tichit, D., Lerner, D. A., \& Marcotte, N. (2009). Azoic dye hosted in layered double hydroxide: Physicochemical characterization of the intercalated materials. Langmuir, 25(18), 10980-10986. doi:10.1021/la901201s

[2] Azam, A., \& Hamid, A. (2006). Effects of gap size and UV dosage on decolorization of CI Acid Orange 7 by UV/H2O2 process. Journal of hazardous materials, 133(1-3), 167-171. doi:10.1016/j.jhazmat.2005.10.005

[3] Zhao, Y., Pan, F., Li, H., Niu, T., Xu, G., \& Chen, W. (2013). Facile synthesis of uniform $\alpha-\mathrm{Fe}_{2} \mathrm{O}_{3}$ crystals and their facet-dependent catalytic performance in the photo-Fenton reaction. Journal of Materials Chemistry A, 1(24), 7242-7246. doi:10.1039/C3TA10966F

[4] Chu, P. (1973). U.S. Patent No. 3,709,979. Washington, DC: U.S. Patent and Trademark Office.

[5] Espinosa, J. C., Catalá, C., Navalón, S., Ferrer, B., Álvaro, M., \& García, H. (2018). Iron oxide nanoparticles supported on diamond nanoparticles as efficient and stable catalyst for the visible light assisted Fenton reaction. Applied Catalysis B: Environmental, 226, 242-251.

[6] Kumar, M. S., Schwidder, M., Grünert, W., \& Brückner, A. (2004). On the nature of different iron sites and their catalytic role in Fe-ZSM-5 DeNOx catalysts: new insights by a combined EPR and UV/VIS spectroscopic approach. Journal of catalysis, 227(2), 384-397. doi: 10.1016/j.jcat.2004.08.003

[7] Pérez-Ramírez, J., Kumar, M. S., \& Brückner, A. (2004). Reduction of N2O with CO over FeMFI zeolites: influence of the preparation method on the iron species and catalytic behavior. Journal of Catalysis, 223(1), 1327. doi: $10.1016 /$ j.jcat.2004.01.007

[8] Messi, C., Carniti, P., \& Gervasini, A. (2008). Kinetics of reduction of supported nanoparticles of iron oxide. Journal of Thermal Analysis and Calorimetry, 91(1), 93-100. doi: 10.1007/s10973-007-8427-7

[9] Pérez-Ramírez, J., Mul, G., Kapteijn, F., Moulijn, J. A., Overweg, A. R., Doménech, A., ... \& Arends, I. W. C. E. (2002). Physicochemical characterization of isomorphously substituted FeZSM-5 during activation. Journal of Catalysis, 207(1), 113-126. doi: 10.1006/jcat.2002.3511

[10] Lin, H. Y., Chen, Y. W., \& Li, C. (2003). The mechanism of reduction of iron oxide by hydrogen. Thermochimica Acta, 400(1-2), 61-67. doi: 10.1016/S0040-6031(02)00478-1

[11] Virkutyte, J., Baruwati, B., \& Varma, R. S. (2010). Visible light induced photobleaching of methylene blue over melamine-doped TiO 2 nanocatalyst. Nanoscale, 2(7), 1109-1111. doi: 10.1039/C0NR00089B

[12] Bordiga, S., Buzzoni, R., Geobaldo, F., Lamberti, C., Giamello, E., Zecchina, A., ... \& Vlaic, G. (1996). Structure and reactivity of framework and extraframework iron in Fe-silicalite as investigated by spectroscopic and physicochemical methods. Journal of Catalysis, 158(2), 486-501. doi: 10.1006/jcat.1996.0048

[13] Heredia, A., Avila, J. G., Vinuesa, A., Saux, C., Mendoza, S. M., Garay, F., \& Crivello, M. (2019). Compared arsenic removal from aqueous solutions by synthetic mixed oxides and modified natural zeolites. Adsorption, 1-12. doi: 10.1007_s10450-019-00109-2 